\title{
From Superstrings to M Theory
}

\author{
John H. Schwarz* \\ ${ }^{*}$ California Institute of Technology ${ }^{1}$ \\ Pasadena, California 91125
}

\begin{abstract}
In this talk I will survey some of the basic facts about superstring theories in 10 dimensions and the dualities that relate them to $\mathrm{M}$ theory in 11 dimensions. Then I will mention some important unresolved issues.
\end{abstract}

\section{INTRODUCTION}

Superstring theory first achieved widespread acceptance during the first superstring revolution in 1984-85. There were three main developments at this time. The first was the discovery of an anomaly cancellation mechanism [2], which showed that supersymmetric gauge theories can be consistent in ten dimensions provided they are coupled to supergravity (as in type I superstring theory) and the gauge group is either $\mathrm{SO}(32)$ or $E_{8} \times E_{8}$. Any other group necessarily would give uncanceled gauge anomalies and hence inconsistency at the quantum level. The second development was the discovery of two new superstring theories - called heterotic string theories - with precisely these gauge groups [3]. The third development was the realization that the $E_{8} \times E_{8}$ heterotic string theory admits solutions in which six of the space dimensions form a Calabi-Yau space, and that this results in a $4 \mathrm{~d}$ effective theory at low energies with many qualitatively realistic features [4]. Unfortunately, there are very many Calabi-Yau spaces and a whole range of additional choices that can be made (orbifolds, Wilson loops, etc.). Thus there is an enormous variety of possibilities, none of which stands out as particularly special.

In any case, after the first superstring revolution subsided, we had five distinct superstring theories with consistent weak coupling perturbation expansions, each in ten dimensions. Three of them, the type $I$ theory and the two heterotic theories, have $\mathcal{N}=1$ supersymmetry in the ten-dimensional sense. Since the minimal 10d spinor is simultaneously Majorana and Weyl, this corresponds to 16 conserved supercharges. The other two theories, called type IIA and type IIB, have $\mathcal{N}=2$ supersymmetry (32 supercharges) [5]. In the IIA case the two spinors have opposite handedness so that the spectrum is left-right symmetric (nonchiral). In the IIB case the two spinors have the same handedness and the spectrum is chiral.

1) Work supported in part by the U.S. Dept. of Energy under Grant No. DE-FG03-92-ER40701. 
The understanding of these five superstring theories was developed in the ensuing years. In each case it became clear, and was largely proved, that there are consistent perturbation expansions of on-shell scattering amplitudes. In four of the five cases (heterotic and type II) the fundamental strings are oriented and unbreakable. As a result, these theories have particularly simple perturbation expansions. Specifically, there is a unique Feynman diagram at each order of the loop expansion. The Feynman diagrams depict string world sheets, and therefore they are two-dimensional surfaces. For these four theories the unique $L$-loop diagram is a closed orientable genus- $L$ Riemann surface, which can be visualized as a sphere with $L$ handles. External (incoming or outgoing) particles are represented by $N$ points (or "punctures") on the Riemann surface. A given diagram represents a well-defined integral of dimension $6 L+2 N-6$. This integral has no ultraviolet divergences, even though the spectrum contains states of arbitrarily high spin (including a massless graviton). From the viewpoint of point-particle contributions, string and supersymmetry properties are responsible for incredible cancellations. Type I superstrings are unoriented and breakable. As a result, the perturbation expansion is more complicated for this theory, and the various world-sheet diagrams at a given order (determined by the Euler number) have to be combined properly to cancel divergences and anomalies [6].

\section{THEORY}

In the 1970s and 1980s various supersymmetry and supergravity theories were constructed. (See [7], for example.) In particular, supersymmetry representation theory showed that ten is the largest spacetime dimension in which there can be a supersymmetric Yang-Mills theory, with spins $\leq 1$ [8]. This is a pretty (i.e., very symmetrical) classical field theory, but at the quantum level it is both nonrenormalizable and anomalous for any nonabelian gauge group. However, as we indicated earlier, both problems can be overcome for suitable gauge groups $(\mathrm{SO}(32)$ or $\left.E_{8} \times E_{8}\right)$ when the Yang-Mills theory is embedded in a type I or heterotic string theory.

The largest possible spacetime dimension for a supergravity theory (with spins $\leq 2$ ), on the other hand, is eleven. Eleven-dimensional supergravity, which has 32 conserved supercharges, was constructed 20 years ago [9]. It has three kinds of fields - the graviton field (with 44 polarizations), the gravitino field (with 128 polarizations), and a three-index antisymmetric tensor gauge field $C_{\mu \nu \rho}$ (with 84 polarizations). These massless particles are referred to collectively as the supergraviton. 11d supergravity is also a pretty classical field theory, which has attracted a lot of attention over the years. It is not chiral, and therefore not subject to anomaly problems. ${ }^{2}$ It is also nonrenormalizable, and thus it cannot be a fundamental theory. However, we now believe that it is a low-energy effective description of $\mathrm{M}$

2) Unless the spacetime has boundaries. The anomaly associated to a $10 \mathrm{~d}$ boundary can be canceled by introducing $E_{8}$ supersymmetric gauge theory on the boundary [10]. 
theory, which is a well-defined quantum theory [11]. This means, in particular, that higher dimension terms in the effective action for the supergravity fields have uniquely determined coefficients within the M theory setting, even though they are formally infinite (and hence undetermined) within the supergravity context.

Intriguing connections between type IIA string theory and $11 \mathrm{~d}$ supergravity have been known for a long time. If one carries out dimensional reduction of $11 \mathrm{~d}$ supergravity to $10 \mathrm{~d}$, one gets type IIA supergravity [12]. In this case dimensional reduction can be viewed as a compactification on a circle in which one drops all the Kaluza-Klein excitations. It is easy to show that this does not break any of the supersymmetries. The field equations of $11 \mathrm{~d}$ supergravity admit a solution that describes a supermembrane. This solution has the property that the energy density is concentrated on a two-dimensional surface. A 3d world-volume description of the dynamics of this supermembrane, quite analogous to the $2 \mathrm{~d}$ world volume actions of superstrings, has been constructed [13]. The authors suggested that a consistent $11 \mathrm{~d}$ quantum theory might be defined in terms of this membrane, in analogy to string theories in ten dimensions. ${ }^{3}$ Another striking result was the discovery of double dimensional reduction [14]. This is a dimensional reduction on a circle, in which one wraps one dimension of the membrane around the circle and drops all Kaluza-Klein excitations for both the spacetime theory and the world-volume theory. The remarkable fact is that this gives the (previously known) type IIA superstring world-volume action [15].

For many years these facts remained unexplained curiosities until they were reconsidered by Townsend [16] and by Witten [11]. The conclusion is that type IIA superstring theory really does have a circular 11th dimension in addition to the previously known ten spacetime dimensions. This fact was not recognized earlier because the appearance of the 11th dimension is a nonperturbative phenomenon, not visible in perturbation theory.

To explain the relation between $M$ theory and type IIA string theory, a good approach is to identify the parameters that characterize each of them and to explain how they are related. Eleven-dimensional supergravity (and hence $M$ theory, too) has no dimensionless parameters. As we have seen, there are no massless scalar fields, whose vevs could give parameters. The only parameter is the $11 \mathrm{~d}$ Newton constant, which raised to a suitable power $(-1 / 9)$, gives the $11 \mathrm{~d}$ Planck mass $m_{p}$. When $\mathrm{M}$ theory is compactified on a circle (so that the spacetime geometry is $R^{10} \times S^{1}$ ) another parameter is the radius $R$ of the circle. The parameters of type IIA superstring theory are the string mass scale $m_{s}$, introduced earlier, and the dimensionless string coupling constant $g_{s}$. An important fact about all five superstring theories is that the coupling constant is not an arbitrary parameter. Rather, it is a dynamically determined vev of a scalar field, the dilaton, which is a supersymmetry partner of the graviton. With the usual conventions, one has $g_{s}=\left\langle e^{\phi}\right\rangle$.

3) It is now clear that this cannot be done in any straightforward manner, since there is no weak coupling limit in which the supermembrane describes all the finite-mass excitations. 
We can identify compactified M theory with type IIA superstring theory by making the following correspondences:

$$
\begin{aligned}
& m_{s}^{2}=2 \pi R m_{p}^{3} \\
& g_{s}=2 \pi R m_{s} .
\end{aligned}
$$

Conventional string perturbation theory is an expansion in powers of $g_{s}$ at fixed $m_{s}$. Equation (2) shows that this is equivalent to an expansion about $R=0$. In particular, the strong coupling limit of type IIA superstring theory corresponds to decompactification of the eleventh dimension, so in a sense M theory is type IIA string theory at infinite coupling. ${ }^{4}$ This explains why the eleventh dimension was not discovered in studies of string perturbation theory.

These relations encode some interesting facts. The fact relevant to eq. (1) concerns the interpretation of the fundamental type IIA string. Earlier we discussed the old notion of double dimensional reduction, which allowed one to derive the IIA superstring world-sheet action from the 11d supermembrane (or M2-brane) world-volume action. Now we can make a stronger statement: The fundamental IIA string actually is an M2-brane of M theory with one of its dimensions wrapped around the circular spatial dimension. No truncation to zero modes is required. Denoting the string and membrane tensions (energy per unit volume) by $T_{F 1}$ and $T_{M 2}$, one deduces that

$$
T_{F 1}=2 \pi R T_{M 2} .
$$

However, $T_{F 1}=2 \pi m_{s}^{2}$ and $T_{M 2}=2 \pi m_{p}^{3}$. Combining these relations gives eq. (1). It should be emphasized that all the formulas in this section are exact, due to the large amount of unbroken supersymmetry.

Type II superstring theories contain a variety of $p$-brane solutions that preserve half of the 32 supersymmetries. These are solutions in which the energy is concentrated on a $p$-dimensional spatial hypersurface. (Adding the time dimension, the world volume of a $p$-brane has $p+1$ dimensions.) The corresponding solutions of supergravity theories were constructed by Horowitz and Strominger [17]. A large class of these $p$-brane excitations are called $D$-branes (or $\mathrm{D} p$-branes when we want to specify the dimension), whose tensions are given by [18]

$$
T_{D p}=2 \pi m_{s}^{p+1} / g_{s} .
$$

This dependence on the coupling constant is one of the characteristic features of a D-brane. It is to be contrasted with the more familiar $g^{-2}$ dependence of soliton masses (e.g., the 't Hooft-Polyakov monopole). Another characteristic feature of D-branes is that they carry a charge that couples to a gauge field in the RamondRamond (RR) sector of the theory. (Such fields can be described as bispinors.)

4) The $E_{8} \times E_{8}$ heterotic string theory is also eleven-dimensional at strong coupling [10]. 
The particular RR gauge fields that occur imply that even values of $p$ occur in the IIA theory and odd values in the IIB theory.

D-branes have a number of special properties, which make them especially interesting. By definition, they are branes on which strings can end-D stands for Dirichlet boundary conditions. The end of a string carries a charge, and the D-brane world-volume theory contains a $U(1)$ gauge field that carries the associated flux. When $n \mathrm{D} p$-branes are coincident, or parallel and nearly coincident, the associated $(p+1)$-dimensional world-volume theory is a $U(n)$ gauge theory. The $n^{2}$ gauge bosons $A_{\mu}^{i j}$ and their supersymmetry partners arise as the ground states of oriented strings running from the $i$ th $\mathrm{D} p$-brane to the $j$ th $\mathrm{D} p$-brane. The diagonal elements, belonging to the Cartan subalgebra, are massless. The field $A_{\mu}^{i j}$ with $i \neq j$ has a mass proportional to the separation of the $i$ th and $j$ th branes. This separation is described by the vev of a corresponding scalar field in the world-volume theory.

In particular, the D2-brane of the type IIA theory corresponds to our friend the supermembrane of $\mathrm{M}$ theory, but now in a background geometry in which one of the transverse dimensions is a circle. The tensions check, because (using eqs. (1), (2), and (4)) $T_{D 2}=2 \pi m_{s}^{3} / g_{s}=2 \pi m_{p}^{3}=T_{M 2}$. The mass of the first Kaluza-Klein excitation of the $11 \mathrm{~d}$ supergraviton is $1 / R$. Using eq. (2), we see that this can be identified with the D0-brane. More identifications of this type arise when we consider the magnetic dual of the $\mathrm{M}$ theory supermembrane. This turns out to be a five-brane, called the M5-brane. ${ }^{5}$ Its tension is $T_{M 5}=2 \pi m_{p}^{6}$. Wrapping one of its dimensions around the circle gives the D4-brane, with tension $T_{D 4}=2 \pi R T_{M 5}=2 \pi m_{s}^{5} / g_{s}$. If, on the other hand, the M5-frame is not wrapped around the circle, one obtains the so-called NS5-brane of the IIA theory with tension

$$
T_{N S 5}=T_{M 5}=2 \pi m_{s}^{6} / g_{s}^{2} .
$$

This 5-brane, which is the magnetic dual of the fundamental IIA string, exhibits the conventional $g^{-2}$ solitonic dependence.

To summarize, type IIA superstring theory is M theory compactified on a circle of radius $R=g_{s} \ell_{s}$. M theory is believed to be a well-defined quantum theory in $11 \mathrm{~d}$, which is approximated at low energy by $11 \mathrm{~d}$ supergravity. Its supersymmetric excitations (which are the only ones known when there is no compactification) are the massless supergraviton, the M2-brane, and the M5-brane. These account both for the (perturbative) fundamental string of the IIA theory and for many of its nonperturbative excitations. The identities presented here are exact, because they are protected by supersymmetry.

\section{IMPORTANT UNRESOLVED ISSUES}

One issue that needs to be settled if superstring theory is to be used for phenomenology is where supersymmetry fits into the story. It is clear that at the string

5) In general, the magnetic dual of a $p$-brane in $d$ dimensions is a $(d-p-4)$-brane. 
scale $\left(\approx 10^{18} \mathrm{GeV}\right)$ or the Planck scale the underlying theory has maximal supersymmetry (32 conserved supercharges). The question that needs to be answered is at what scales they are broken and by what mechanisms. The traditional picture (which looks the most plausible to me) is that at the compactification/GUT scale $\left(\approx 10^{16} \mathrm{GeV}\right)$ the symmetry is broken to $\mathcal{N}=1$ in $d=4$ (four conserved supercharges), and this persists to the $\mathrm{TeV}$ scale, where the final susy breaking occurs. The TeV scale is indicated by three separate arguments: the gauge hierarchy problem, supersymmetric grand unification, and the requirement that the lightest superparticle (LSP) be a cosmologically significant component of dark matter. It would be astonishing if this coincidence turned out to be a fluke. There is other support for this picture such as the mass of the top quark and the ease with which it gives electroweak symmetry breaking. Despite all these indications, we cannot be certain that this picture is correct until it is demonstrated experimentally. As I once told a newspaper reporter: discovery of supersymmetry would be more profound than life on Mars.

Another important issue is the problem of vacuum degeneracy and the stabilization of moduli. Let me explain. The underlying theory is completely unique, with no dimensionless parameters. Nevertheless, typical quantum vacua have continuous parameters, called moduli, which arise as the vacuum values of scalar fields. Notable examples are the sizes of extra dimensions and the string coupling constant. For typical string vacua, the effective potential has many flat directions, so there is a continuum, or moduli space, of minima. The fields that correspond to the flat directions describe massless spin zero particles. These particles typically interact with roughly gravitational strength, which is a problem because the gravitational force is observed to be pure tensor to better than $1 \%$ accuracy. So it seems that we should seek a vacuum without moduli, which is very difficult to do. However, if a realistic vacuum of this type is ever found, it will not have any continuously adjustable parameters, and therefore it will be completely predictive (at least in principle).

Perhaps the most challenging unresolved issue of all, is the cosmological constant. This is a term in the effective action that describes the energy density of the vacuum, which is observable in a gravitational theory. Observationally, there are indications that it may be nonzero, but it is extremely small. Taking the $1 / 4$ power, the energy scale is $\leq 10^{-11} \mathrm{GeV}$. In a fundamental theory it receives contributions from many sources such as vacuum condensates and zero point energies. Supersymmetry ensures that boson and fermion zero point energies cancel, so the natural scale would seem to be the TeV susy breaking scale, which is many orders of magnitude too high. This is a fine-tuning problem that is reminiscent of the gauge hierarchy problem. Presumably string theory will provide an elegant solution. Until we know what the relevant mechanism is, it is hard to be confident that there is not an alternative to supersymmetry for solving the gauge hierarchy problem. I believe that when the correct solution to the problem of the cosmological constant is found, it will spark another revolution in our understanding. Recently toy models without supersymmetry that seem to have a vanishing cosmological constant have been 
constructed by Kachru, Kumar, and Silverstein [19]. These models are far from realistic, and have not led yet to new qualitative understandings. However, they are the best lead we have at the present time.

To conclude, there has been dramatic progress in understanding string theory in the past few years, but there are still crucial issues that remain unresolved. Future experimental discoveries will be essential to help guide our thinking. Sooner (Tevatron) or later (LHC) exciting new phenomena are bound to show up. My bet is on Higgs and superparticles. But if I should turn out to be wrong, that would not mean that string theory is wrong.

\section{REFERENCES}

1. Schwarz, J.H., hep-th/9807135.

2. Green, M.B., and Schwarz, J.H., Phys. Lett. 149B, 117 (1984).

3. Gross, D.J., Harvey, J.A., Martinec, E., and Rohm, R., Phys. Rev. Lett. 54, 502 (1985).

4. Candelas, P., Horowitz, G.T., Strominger, A., and Witten, E., Nucl. Phys. B258, 46 (1985).

5. Green, M.B., and Schwarz, J.H., Phys. Lett. 109B, 444 (1982).

6. Green, M.B., and Schwarz, J.H., Phys. Lett. 151B, 21 (1985); Nucl. Phys. B255, 93 (1985).

7. Salam, A., and Sezgin, E., eds., Supergravities in Diverse Dimensions, reprints in 2 vols., World Scientific (1989).

8. Brink, L., Schwarz, J.H., and Scherk, J., Nucl. Phys. B121, 77(1977); Gliozzi, F., Scherk, J., and Olive, D., Nucl. Phys. B122, 253 (1977).

9. Cremmer, E., Julia, B., and Scherk, J., Phys. Lett. 76B, 409 (1978).

10. Hořava, P., and Witten, E., Nucl. Phys. B460, 506 (1996), hep-th/9510209.

11. Witten, E., Nucl. Phys. B443, 85 (1995), hep-th/9503124.

12. Campbell. C., and West, P., Nucl. Phys. B243, 112 (1984); Huq, M., and Namazie, M., Class. Quantum Grav. 2, 293 (1985); Giani, F., and Pernici, M., Phys. Rev. D30, 325 (1984).

13. Bergshoeff, E., Sezgin, E., and Townsend, P.K., Phys. Lett. B189, 75 (1987).

14. Duff, M.J., Howe, P.S., Inami, T., and Stelle, K.S., Phys. Lett. B191, 70 (1987).

15. Green, M.B., and Schwarz, J.H., Phys. Lett. 136B, 367 (1984).

16. Townsend, P.K., Phys. Lett. B350, 184 (1995), hep-th/9501068.

17. Horowitz, G.T., and Strominger, A., Nucl. Phys., 197 B360 (1991).

18. Polchinski, J., Phys. Rev. Lett. 75, 4724 (1995), hep-th/9510017; p. 293 in Fields, Strings, and Duality (TASI 96), eds. C. Efthimiou and B. Greene, World Scientific 1997, hep-th/9611050.

19. Kachru, S., Kumar, J., and Silverstein, E., hep-th/9807076. 